\title{
Gene expression profile analysis of colorectal cancer to investigate potential mechanisms using bioinformatics
}

This article was published in the following Dove Press journal:

OncoTargets and Therapy

8 April 2015

Number of times this article has been viewed

Yubin Kou ${ }^{1,2, *}$

Suya Zhang ${ }^{3, *}$

Xiaoping Chen ${ }^{2}$

Sanyuan $\mathrm{Hu}^{\prime}$

'Department of General Surgery, Qilu Hospital of Shandong University, Jinan, People's Republic of China; ${ }^{2}$ Department of General Surgery, ${ }^{3}$ Department of Neurology, Shuguang Hospital Baoshan Branch, Shanghai,

People's Republic of China

*These authors contributed equally to this work
Correspondence: Sanyuan Hu Department of General Surgery, Qilu Hospital of Shandong University, No 107 Wenhua Xi Road, Jinan 2500I2, People's Republic of China

Tel +8653182169114

Fax +8653186927544

Email husanyuan00I@163.com
Abstract: This study aimed to explore the underlying molecular mechanisms of colorectal cancer (CRC) using bioinformatics analysis. Using GSE4107 datasets downloaded from the Gene Expression Omnibus, the differentially expressed genes (DEGs) were screened by comparing the RNA expression from the colonic mucosa between $12 \mathrm{CRC}$ patients and ten healthy controls using a paired $t$-test. The Gene Ontology (GO) functional and pathway enrichment analyses of DEGs were performed using the Database for Annotation, Visualization and Integrated Discovery (DAVID) software followed by the construction of a protein-protein interaction (PPI) network. In addition, hub gene identification and GO functional and pathway enrichment analyses of the modules were performed. A total of 612 up- and 639 downregulated genes were identified. The upregulated DEGs were mainly involved in the regulation of cell growth, migration, and the MAPK signaling pathway. The downregulated DEGs were significantly associated with oxidative phosphorylation, Alzheimer's disease, and Parkinson's disease. Moreover, FOS, $F N 1, P P P 1 C C$, and CYP2B6 were selected as hub genes in the PPI networks. Two modules (up-A and up-B) in the upregulated PPI network and three modules (d-A, d-B, and d-C) in the downregulated PPI were identified with the threshold of Molecular Complex Detection (MCODE) Molecular Complex Detection (MCODE) score $\geq 4$ and nodes $\geq 6$. The genes in module up-A were significantly enriched in neuroactive ligand-receptor interactions and the calcium signaling pathway. The genes in module d-A were enriched in four pathways, including oxidative phosphorylation and Parkinson's disease. DEGs, such as FOS, FN1, PPP1CC, and CYP2B6, may be used as potential targets for CRC diagnosis and treatment.

Keywords: molecular mechanisms, network module, enrichment analysis

\section{Introduction}

Colorectal cancer (CRC) is a leading cause of cancer-related deaths worldwide, accounting for more than 1 million cases and 600,000 deaths every year. ${ }^{1}$ CRC is a cancer mostly due to uncontrolled cell growth in the colon, rectum, or appendix. Many factors may cause CRC including smoking, obesity, alcohol, older age, high fat intake, and a lack of physical exercise. ${ }^{2}$ For patients in the age range of 55-64 years, flexible sigmoidoscopic screening has been used to treat CRC and could reduce its incidence and mortality by $33 \%$ and $43 \%$, respectively. ${ }^{3}$ Although colonoscopic screening has become a reliable screening tool for treatment of CRC, it has the shortcomings of bleeding and perforations, as well as a tendency to cause acute diverticulitis. ${ }^{4}$ Therefore, the identification of reliable, accurate, and sensitive biomarkers to improve the detection of CRC is imperative.

Over the past decades, various genetic studies have been established, which are associated with the accumulated process and progress of CRC. For example, 
a recent study identified that the microRNA-126 that targets CXCR4 was downregulated in CRC tissue and was involved in distant metastasis, clinical tumornode-metastasis staging, and poor survival. ${ }^{5} \mathrm{Xu}$ et $\mathrm{al}^{6}$ reported that downregulated microRNA-375 in tissue and plasma was matched in CRC and associated with some critical signal pathways, such as MAPK, Wnt, and TGF-beta signal pathways in the development and progression of CRC. In addition, based on gene/exon/network-level analysis tools, $A T P 8 B 1$ was found to be a novel gene associated with CRC, which changed at the cytogenetic, gene, and exon levels. ${ }^{7}$ After implementing a computational compilation of protein conformational changes and the probable CRC-associated phenotype, Kumar et $\mathrm{al}^{8}$ found that the E403K mutation in the mitotic centromere-associated kinesis protein could produce CRC-associated phenotypic affects by producing a major impact on the structural conformation of the protein. Recent evidence generated from an experimental interleukin-10deficient mouse model using microbial genomic analysis showed that inflammation played an important role in bacteria-induced CRC. ${ }^{9}$ Although these expanded efforts studied the genetic bases of CRC, the molecular mechanisms of the development and progress are not fully understood.

Recently, gene expression profile data associated with CRC have been studied by many researchers. For example, Liu et $\mathrm{al}^{10}$ downloaded the microarray data of GSE4107 and GSE8671, screened common differentially expressed genes (DEGs) between CRC and healthy controls (HC), and performed Gene Ontology (GO) functional and Kyoto Encyclopedia of Genes and Genomes (KEGG) pathway enrichment analyses. However, they did not perform further study of DEGs, such as the construction of a protein-protein interaction (PPI) network and module analysis of the PPI network.

In the present study, using the same microarray data (GSE4107), we compared RNA expression from colonic mucosa between 12 patients with CRC and ten HC samples to identify the DEGs. In addition, we constructed the PPI network of the DEGs and performed hub gene identification and module analysis of the PPI network. The findings of this study may provide an improved understanding of and lead to an improved diagnosis of CRC.

\section{Materials and methods Affymetrix chip data}

The Gene Expression Omnibus (GEO) (http://www.ncbi.nlm. nih.gov/geo/) database at the National Center for Biotechnology Information (NCBI) is currently the largest fully public gene expression resource, which includes 214,268 samples and 4,500 platforms. ${ }^{11}$ The chip dataset GSE4107, which included $12 \mathrm{CRC}$ patients and ten $\mathrm{HC}$ samples, was downloaded from GEO. ${ }^{12}$ The HC and CRC samples were age- (50 years or less), ethnicity- (Chinese), and tissue-matched. The RNA extracted from the colonic mucosa of the HC and CRC samples was analyzed using the GPL570 (HG-U133_Plus_2) Affymetrix Human Genome U133 Plus 2.0 Array.

\section{Data preprocessing and analysis of DEGs}

The affy package is an $\mathrm{R}$ package of functions and classes for the analysis of oligonucleotide arrays. ${ }^{13}$ Data preprocessing was performed using robust multi-array average in the affy package in $\mathrm{R}$, including background adjustment, normalization, and $\log$ transformation of the values. ${ }^{14}$ Then, the original CEL file data were transformed to probe-level data. Further, the probe-level data were converted to gene symbols using the Perl procedure given that when several probes corresponded to one gene symbol, the gene expression value was the mean of the probes. Multiple testing was corrected using the Benjamini-Hochberg ${ }^{15}$ procedure to obtain the adjusted $P$-value. Then, the $\log 2$-fold change $(\log 2 \mathrm{FC})$ was calculated. Only genes with an adjusted $P$-value $<0.05$ and $|\log 2 \mathrm{FC}|>1$ were regarded as DEGs.

\section{Functional and pathway enrichment analysis}

As a comprehensive set of functional annotation tools, the Database for Annotation, Visualization and Integrated Discovery (DAVID) has been used for systematic and integrative analysis of large gene lists. ${ }^{16} \mathrm{GO}$ terms are significantly overrepresented in a set of genes from three aspects, including the cellular component, molecular function, and biological process. ${ }^{17}$ In our work, the significant GO biological process terms and KEGG pathway enrichment analyses of the identified DEGs were performed using DAVID with the thresholds of $P$-value $<0.05$ and enrichment gene count $>2$.

\section{PPI network construction}

The Search Tool for the Retrieval of Interacting Genes (STRING) database (http://www.bork.embl-heidelberg.de/ $\underline{\text { STRING//) }})^{18}$ is a pre-computed global resource for the exploration and analysis of PPI information. In the present study, the STRING 9.0 online tool was used to screen the PPI of the DEGs. The DEGs with required confidence (combined score) $>0.4$ were selected, and then the PPI network was constructed and visualized using Cytoscape. ${ }^{19}$ Given that 
most of the networks were scale-free, the hub genes were then selected with a connectivity degree $>10$.

\section{Module analysis of the PPI network}

The module analysis was performed on the PPI network using the Molecular Complex Detection (MCODE) in Cytoscape with a degree cutoff $=2$, node score cutoff $=0.2$, $\mathrm{k}$-core $=2$, and max depth $=100 .{ }^{20}$ Then, the significant modules with MCODE score $\geq 4$ and node $\geq 6$ were selected. Next, GO functional and KEGG pathway enrichment analyses of the most significant module were performed with a threshold of $P<0.05$.

\section{Results Identification of DEGs}

Using bioinformatics approaches, a total of 1,251 DEGs between ten $\mathrm{HC}$ and 12 CRC samples were identified, including 612 upregulated and 639 downregulated genes.

\section{GO functional and pathway enrichment analysis}

Based on DAVID software, a total of $29 \mathrm{GO}$ functions were enriched. Among them, the upregulated DEGs were mainly enriched in 15 GO functions and the downregulated DEGs were mainly enriched in $14 \mathrm{GO}$ functions. The top five GO terms in each are shown in Table 1. The most significant GO function in the upregulated DEGs was the regulation of cell growth, and the most significant one in the downregulated DEGs was Golgi vesicle transport.

After KEGG pathway enrichment analysis, the upregulated DEGs were mainly enriched in seven KEGG pathways including vascular smooth muscle contraction, axon guidance, and the MAPK signaling pathway. The downregulated DEGs were mainly enriched in ten pathways, such as oxidative phosphorylation and Parkinson's disease (Table 2).

\section{PPI network construction and hub gene identification}

After the upregulated and downregulated DEGs were submitted into STRING, a total of 998 upregulated and 900 downregulated PPI relationships were obtained, respectively. Then, the hub genes or proteins in the networks with connectivity degree $>10$ were identified (Table 3 ). A total of 44 hub genes were selected from the upregulated PPI network, which included FBJ murine osteosarcoma viral oncogene homolog $(F O S)$, fibronectin 1 (FN1), and CREB binding protein $(C R E B B P)$. Meanwhile, 43 hub genes, such as protein phosphatase 1 , catalytic subunit, gamma isozyme (PPPICC); ATP synthase, $\mathrm{H}+$ transporting, mitochondrial Fo complex, subunit B1 (ATP5F1); and cytochrome P450, family 2 , subfamily B, polypeptide 6 (CYP2B6), were identified from the downregulated PPI network.

\section{Module analysis}

Two modules with MCODE scores $\geq 4$ and nodes $\geq 6$ were selected in the upregulated PPI networks: up-A (MCODE score $=6.429)$ with 29 nodes and up-B $($ MCODE score $=4.522)$ with 24 nodes (Figure 1). Meanwhile, there were three modules in the downregulated PPI network: d-A (MCODE score $=14$ ) with 15 nodes, d-B (MCODE score $=7.75$ ) with nine nodes, and d-C (MCODE score =7.714) with eight nodes (Figure 2).

The GO functional enrichment scores of modules up-A and d-A were 3.54 and 15.55, respectively. Module up-A

Table I GO functional enrichment analysis of up- and downregulated DEGs (top five in each)

\begin{tabular}{|c|c|c|c|c|c|}
\hline Category & Term & Description & Count & $P$-value & Size \\
\hline \multicolumn{6}{|c|}{ Upregulated } \\
\hline $\mathrm{BP}$ & GO:000I558 & Regulation of cell growth & 20 & $3.38 \mathrm{E}-04$ & 193 \\
\hline $\mathrm{BP}$ & GO:0016477 & Cell migration & 24 & $6.74 \mathrm{E}-04$ & 270 \\
\hline $\mathrm{BP}$ & GO:0044057 & Regulation of system process & 26 & $7.02 \mathrm{E}-04$ & 305 \\
\hline $\mathrm{BP}$ & GO:0040008 & Regulation of growth & 27 & I.3IE-03 & 336 \\
\hline $\mathrm{BP}$ & GO:000836I & Regulation of cell size & 19 & $\mathrm{I} .8 \mathrm{IE}-03$ & 205 \\
\hline \multicolumn{6}{|c|}{ Downregulated } \\
\hline $\mathrm{BP}$ & GO:0048193 & Golgi vesicle transport & 16 & $3.24 \mathrm{E}-05$ & $|3|$ \\
\hline $\mathrm{BP}$ & GO:0015031 & Protein transport & 45 & $2.18 \mathrm{E}-04$ & 752 \\
\hline $\mathrm{BP}$ & GO:0045I84 & Establishment of protein localization & 45 & 2.63E-04 & 759 \\
\hline $\mathrm{BP}$ & GO:0046907 & Intracellular transport & 40 & $3.21 \mathrm{E}-04$ & 652 \\
\hline BP & GO:0070727 & Cellular macromolecule localization & 28 & $6.28 \mathrm{E}-04$ & 409 \\
\hline
\end{tabular}

Notes: Category: GO function. Count: the number of DEGs. The $P$-value was adjusted using the Benjamini-Hochberg method. Size: the total number of genes in the GO BP. Abbreviations: BP, biological process; DEGs, differentially expressed genes; GO, Gene Ontology. 
Table 2 The enriched KEGG pathway of DEGs

\begin{tabular}{|c|c|c|c|c|c|}
\hline Category & Term & Description & Count & $P$-value & Size \\
\hline \multicolumn{6}{|c|}{ Upregulated } \\
\hline KEGG & hsa04270 & Vascular smooth muscle contraction & 10 & $3.45 \mathrm{E}-02$ & III \\
\hline KEGG & hsa04360 & Axon guidance & 11 & $3.50 \mathrm{E}-02$ & 129 \\
\hline KEGG & hsa040I0 & MAPK signaling pathway & 18 & $3.93 \mathrm{E}-02$ & 266 \\
\hline \multicolumn{6}{|c|}{ Downregulated } \\
\hline KEGG & hsa00I90 & Oxidative phosphorylation & 18 & $6.70 \mathrm{E}-06$ & 119 \\
\hline KEGG & hsa050I2 & Parkinson's disease & 17 & I.59E-05 & 114 \\
\hline KEGG & hsa050I0 & Alzheimer's disease & 19 & $7.04 \mathrm{E}-05$ & 156 \\
\hline KEGG & hsa050I6 & Huntington's disease & 20 & $7.42 \mathrm{E}-05$ & 171 \\
\hline KEGG & hsa00970 & Aminoacyl-tRNA biosynthesis & 8 & I.33E-03 & 41 \\
\hline KEGG & hsa00830 & Retinol metabolism & 8 & $3.83 \mathrm{E}-03$ & 49 \\
\hline KEGG & hsa00860 & Porphyrin and chlorophyll metabolism & 6 & $7.30 \mathrm{E}-03$ & 30 \\
\hline KEGG & hsa00280 & Valine, leucine, and isoleucine degradation & 6 & $3.49 \mathrm{E}-02$ & 44 \\
\hline KEGG & hsa04664 & Fc epsilon RI signaling pathway & 8 & $3.80 \mathrm{E}-02$ & 76 \\
\hline KEGG & hsa04666 & Fc gamma R-mediated phagocytosis & 9 & 4.I3E-02 & 94 \\
\hline
\end{tabular}

Notes: Count: the number of DEGs. Size: the total number of genes in the pathway.

Abbreviations: DEGs, differentially expressed genes; KEGG, Kyoto Encyclopedia of Genes and Genomes.

was enriched in six functions such as behavior, taxis, and chemotaxis; module d-A was enriched in four functions such as oxidative phosphorylation and phosphorus metabolic process (Table 4). In addition, the KEGG pathway analysis revealed that the genes in module up-A were significantly enriched in pathways including neuroactive ligand-receptor interactions and the calcium signaling pathway; the genes in module d-A were significantly enriched in oxidative phosphorylation, Parkinson's disease, Alzheimer's disease, and Huntington's disease (Table 5).

\section{Discussion}

The analysis of gene expression profiling has been widely used to reveal the abnormally expressed genes associated with CRC and has enabled the identification of targets for therapeutic strategies. In our study, the CRC mechanism was analyzed by bioinformatics, including DEGs screening, PPI network construction, hub gene identification, and module analysis of the PPI network. Based on these results, the underlying molecular mechanisms of CRC were explored on genetic and molecular levels, which provided new insights into CRC diagnosis and treatment.

After analyzing the hub genes from the upregulated PPI network, FOS and FN1 were selected with the high connective degree. The FOS gene family was demonstrated to encode leucine zipper proteins and act as a regulator of cell proliferation, differentiation, and transformation. ${ }^{21}$ FOS was upregulated in the mucosa of CRC and participated in the biological processes of transcription regulation, cell growth, and inflammatory response in early-onset CRC. ${ }^{16}$ In addition, the overexpression of Fra-1, a member of the FOS family, played an essential role in tumorigenesis and was required for the motility and invasion of $\mathrm{CRC}$ cells through the downregulation of $\beta 1$-integrin, resulting in decreased

Table 3 The hub proteins in the upregulated and downregulated protein-protein interaction network (top 20 in each)

\begin{tabular}{|c|c|c|c|c|c|c|c|}
\hline ID & Degree & ID & Degree & ID & Degree & ID & Degree \\
\hline \multicolumn{8}{|l|}{ Upregulated } \\
\hline FOS & 41 & IGFI & 25 & DES & 19 & CALDI & 16 \\
\hline FNI & 34 & CD44 & 25 & EGRI & 19 & CAVI & 16 \\
\hline CREBBP & 33 & COLI4AI & 21 & TAGLN & 17 & RET & 16 \\
\hline ESRI & 32 & MGP & 20 & ATF3 & 17 & ACTA2 & 15 \\
\hline $\mathrm{DCN}$ & 26 & GSK3B & 20 & MYHII & 16 & RUNXITI & 15 \\
\hline \multicolumn{8}{|c|}{ Downregulated } \\
\hline PPPICC & 24 & $\mathrm{HDACl}$ & 22 & SOD2 & 20 & ATP5O & 18 \\
\hline ATP5FI & 24 & GAPDH & 22 & PPARA & 19 & $\mathrm{RACl}$ & 17 \\
\hline CYP2B6 & 23 & MAPKI & 22 & UQCRH & 19 & COX4II & 17 \\
\hline ATP5] & 23 & ATP5L & 21 & HINTI & 18 & Cl4orf2 & 16 \\
\hline DHXI5 & 23 & UQCRII & 20 & ATP5G3 & 18 & coX7C & 15 \\
\hline
\end{tabular}

Note: Degree: the connectivity of the protein. 


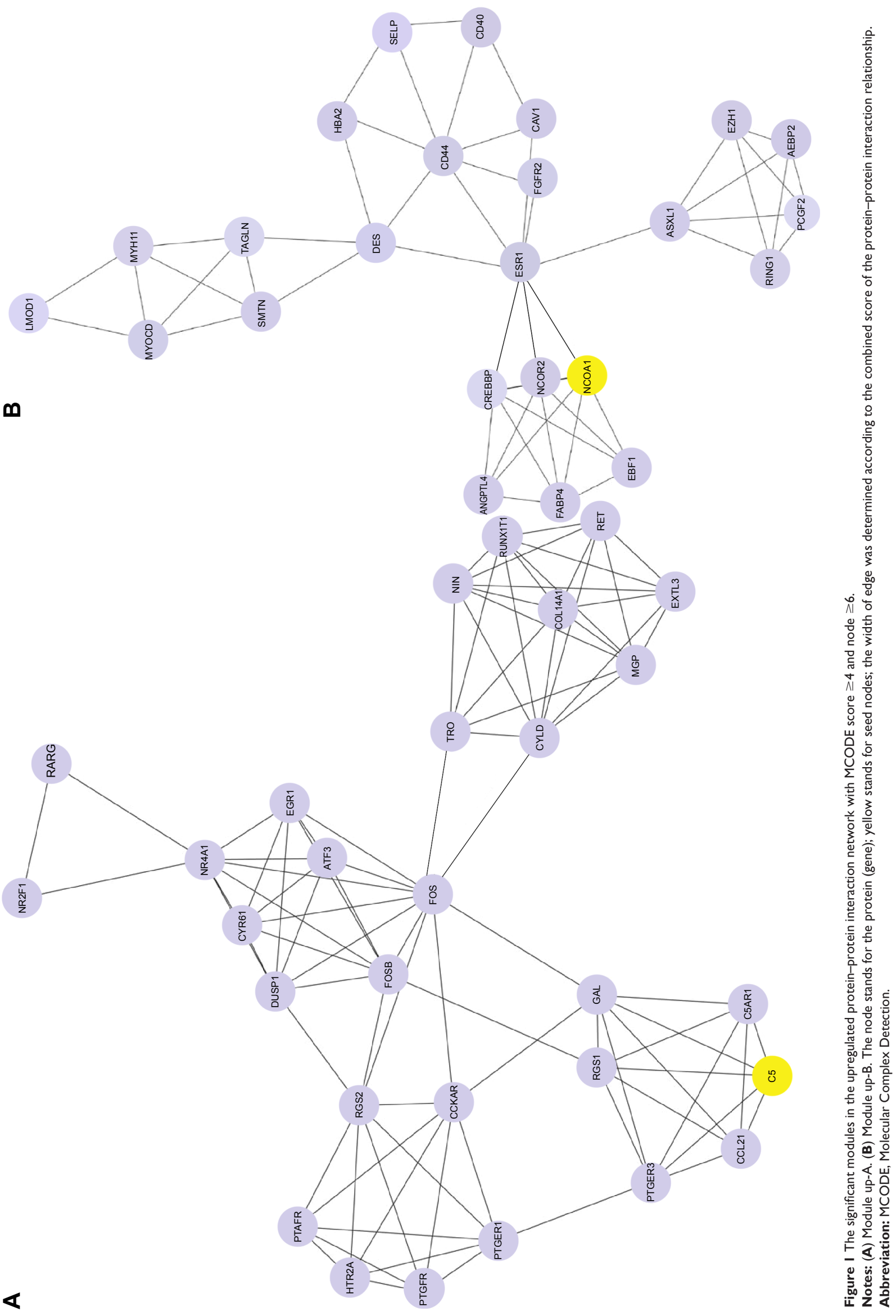



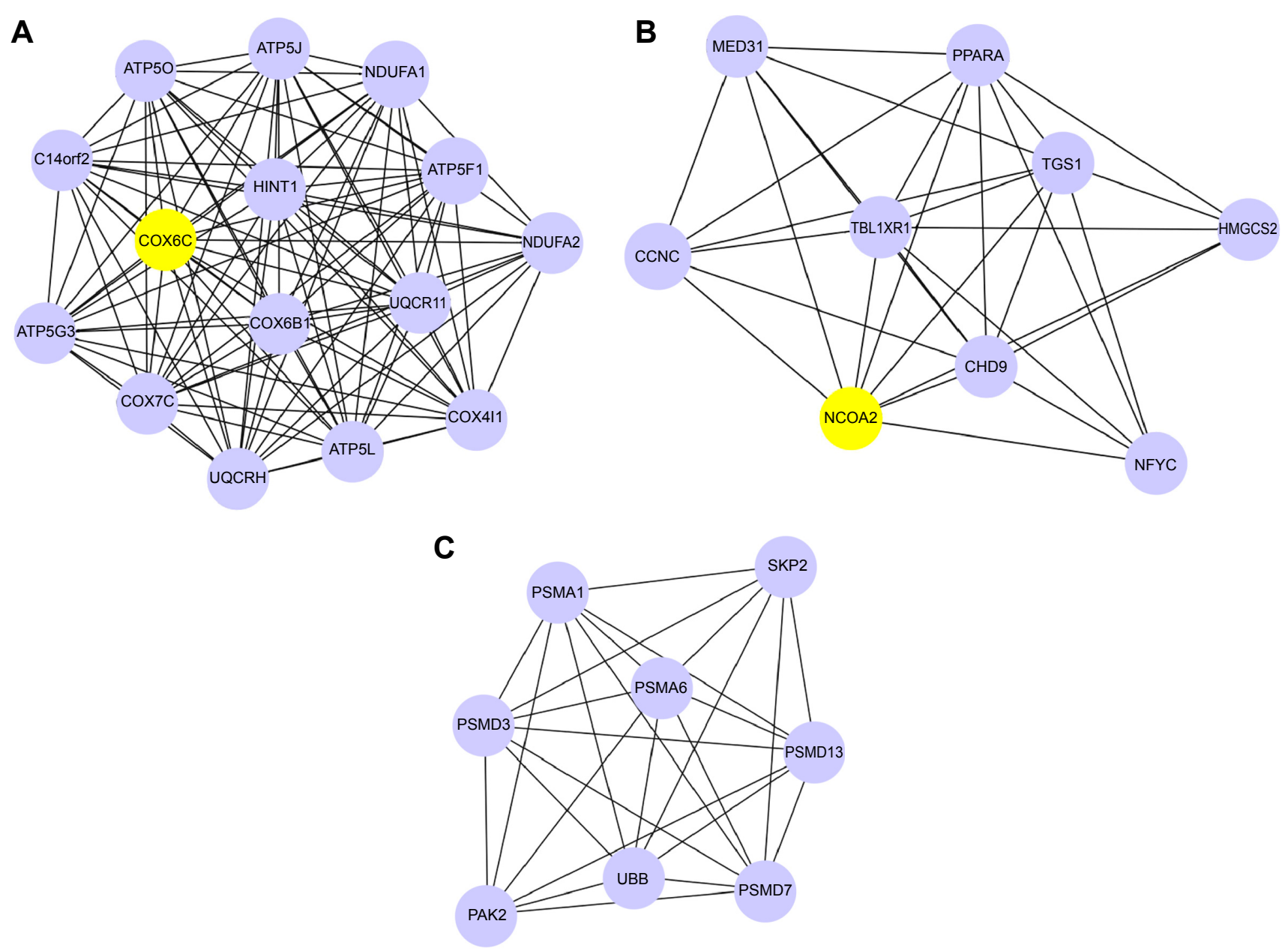

Figure 2 The significant modules in the downregulated protein-protein interaction network with MCODE score $\geq 4$ and node $\geq 6$.

Notes: (A) Module d-A. (B) Module d-B. (C) Module d-C. The node stands for the protein (gene); yellow stands for seed nodes; the width of edge was determined according to the combined score of the protein-protein interaction relationship.

Abbreviation: MCODE, Molecular Complex Detection.

cell-substratum adhesion and cell inactivation of the PhoROCK pathway. 22,23

FN1 has been found to play a major role in cell adhesion, growth, migration, differentiation, and wound healing. ${ }^{24}$ Increased expression of $F N 1$ was found in node-positive CRC and mediated migration of CRC cells in vitro. ${ }^{25}$ Additionally, it is well established that $F N$ expression is involved in the tissue remodeling processes that are associated with CRC progression. ${ }^{26}$ Together with the genes that participate as mediators of cytoskeletal organization and integrin signaling,

Table 4 The GO functional enrichment analysis of DEGs in the up-A and d-A modules with the threshold of $P<0.05$

\begin{tabular}{lllll}
\hline Category & Term & Description & Count & P-value \\
\hline Module up-A & Enrichment score:3.54 & & & Size \\
BP & GO:00076I0 & Behavior & II & $2.57 \mathrm{E}-08$ \\
BP & GO:0042330 & Taxis & 5 & $3.45 \mathrm{E}-04$ \\
BP & GO:0006935 & Chemotaxis & 5 & $3.45 \mathrm{E}-04$ \\
BP & GO:0006952 & Defense response & 154 \\
BP & GO:0007626 & Locomotory behavior & 7 & 154 \\
BP & GO:0006955 & Immune response & 5 & 587 \\
Module d-A & Enrichment score:I5.55 & & 5 & $2.56 \mathrm{E}-03$ \\
BP & GO:0006II9 & Oxidative phosphorylation & 9 & $4.88 \mathrm{E}-02$ \\
BP & GO:0016310 & Phosphorylation & 632 \\
BP & GO:0006793 & Phosphorus metabolic process & 9 & $2.53 \mathrm{E}-15$ \\
BP & GO:0006796 & Phosphate metabolic process & 9 & 8.1 IE-08 \\
\hline
\end{tabular}

Notes: Category: GO function. Count: the number of DEGs. Enrichment score: the score of GO enrichment. Size: the total number of genes in the GO BP. Abbreviations: BP, biological process; DEGs, differentially expressed genes; GO, Gene Ontology. 
Table 5 The KEGG pathway enrichment analysis of DEGs in the up-A and d-A modules with the threshold of $P<0.05$

\begin{tabular}{lllrr}
\hline Category & Term & Description & Count & P-value \\
\hline $\begin{array}{l}\text { Module up-A } \\
\text { Pathway }\end{array}$ & hsa04080 & Neuroactive ligand-receptor interaction & 7 & Size \\
$\begin{array}{l}\text { Pathway } \\
\text { Module d-A }\end{array}$ & hsa04020 & Calcium signaling pathway & 6 & $1.16 \mathrm{E}-04$ \\
$\quad \begin{array}{l}\text { Pathway } \\
\text { Pathway }\end{array}$ & hsa00190 & Oxidative phosphorylation & 13 & $2.07 \mathrm{E}-04$ \\
Pathway & hsa05012 & Parkinson's disease & 12 & $3.75 \mathrm{E}-20$ \\
Pathway & hsa05010 & Alzheimer's disease & 12 & $1.17 \mathrm{E}-17$ \\
\hline
\end{tabular}

Notes: Category: KEGG pathway. Count: the number of DEGs. Size: the total number of genes in the pathway.

Abbreviations: DEGs, differentially expressed genes; KEGG, Kyoto Encyclopedia of Genes and Genomes.

they form a pattern of colon cancer-specific changes and particularly impact cell motility. ${ }^{27}$ In our study, FOS and FN1 were upregulated in the PPI network in CRC, suggesting that FOS and FN1 may be enrolled in the process and progress of CRC. However, further studies are needed to verify our hypothesis.

Additionally, $P P P 1 C C$ and $C Y P 2 B 6$ were found as two hub genes in the downregulated PPI network. PPP1CC belongs to the protein phosphatase family, protein phosphatase 1 (PP1) subfamily. After analyzing the microarray data of colon cancer patients by using a Takagi-Sugeno-Kang (TSK)-type recurrent neural fuzzy network, Vineetha et al ${ }^{28}$ revealed that $P P P 1 C C$ is associated with the GO term of proteolysis involved in cellular protein catabolic process and the insulin signaling pathway in CRC-related canonical pathways. In addition, PP1 was found to play an essential role in negatively regulating the extracellular signal-regulated kinase pathway in CRC cells. ${ }^{29}$ CYP2B6 encodes a member of the cytochrome $\mathrm{P} 450$ superfamily of enzymes. The cytochrome P450 enzymes play an important role in tumor development through their metabolism of many carcinogens. ${ }^{30}$ Several cytochrome $\mathrm{P} 450$ s were found via immunohistochemistry to have increased expression in CRC and may act as independent markers of prognosis. ${ }^{31}$ Compounds associated with the etiology of CRC contain polycyclic aromatic hydrocarbons, in particular, heterocyclic amines, which require metabolic activation by the cytochrome P450 enzymes before exerting their genotoxic effect. ${ }^{32}$ In addition, many of the cytochrome P450 enzyme genes such as CYP1A2 and CYP1B1 play essential roles in CRC susceptibility. ${ }^{33}$ Taken together, these data support the hypothesis that PPP1CC and CYP2B6 may act as candidate molecular markers associated with CRC.

In the current study, our data were downloaded from the gene expression profile dataset GSE4107, which has been reported by Hong et al. ${ }^{12}$ As in Hong et al's study, we also analyzed the DEGs between CRC samples and $\mathrm{HC}$ and performed GO functional and pathway enrichment analyses to explore the potential molecular mechanism of CRC.
However, there are still some discrepancies. Based on Hong et al's study, further studies using bioinformatics were performed, such as the construction of a PPI network, hub gene analysis, and module analysis of the PPI network. Then, 998 upregulated and 900 downregulated PPI relationships were obtained followed by the identification of 44 hub genes from the upregulated PPI network and 43 from the downregulated PPI network. Additionally, two modules (up-A and up-B) in the upregulated PPI network and three modules (d-A, d-B, and d-C) in the downregulated PPI network were identified.

However, there are some limitations in our study. First, the small amount of data used in the current study were downloaded from the GEO database, not generated by us. Because GEO is a huge data repository, a meta-analysis of the relevant datasets of CRC may be performed in future studies. Second, the results were web-based and were not verified by biological experiments. Thus, further experimental studies based on our findings are still needed.

In summary, our study provides a comprehensive bioinformatics analysis of DEGs which may be involved in the progression and development of CRC. The findings of this study may provide a basis for understanding the underlying molecular mechanisms of CRC. In addition, DEGs such as FOS, FNI, PPPICC, and CYP2B6 may be used as potential therapeutic targets for CRC. However, further studies are necessary for improving the diagnosis and treatment of CRC through regulating DEGs.

\section{Disclosure}

The authors report no conflicts of interest in this work.

\section{References}

1. Goel A, Boland CR. Recent insights into the pathogenesis of colorectal cancer. Curr Opin Gastroenterol. 2010;26(1):47-52.

2. Zauber AG, Winawer SJ, O’Brien MJ, et al. Colonoscopic polypectomy and long-term prevention of colorectal-cancer deaths. $N$ Engl J Med. 2012;366(8):687-696.

3. Atkin WS, Edwards R, Kralj-Hans I, et al; UK Flexible Sigmoidoscopy Trial Investigators. Once-only flexible sigmoidoscopy screening in prevention of colorectal cancer: a multicentre randomised controlled trial. Lancet. 2010;375(9726):1624-1633. 
4. RutterCM,JohnsonE, Miglioretti DL, Mandelson MT, Inadomi J,BuistDS. Adverse events after screening and follow-up colonoscopy. Cancer Causes Control. 2012;23(2):289-296.

5. Liu Y, Zhou Y, Feng X, et al. Low expression of microRNA-126 is associated with poor prognosis in colorectal cancer. Genes Chromosomes Cancer. 2014;53(4):358-365.

6. Xu L, Li M, Wang M, Yan D, Feng G, An G. The expression of microRNA-375 in plasma and tissue is matched in human colorectal cancer. BMC Cancer. 2014;14:714.

7. Aziz MA, Periyasamy S, Al Yousef Z, et al. Integrated exon level expression analysis of driver genes explain their role in colorectal cancer. PLoS One. 2014;9(10):e110134.

8. Kumar A, Rajendran V, Sethumadhavan R, Purohit R. Evidence of colorectal cancer-associated mutation in MCAK: a computational report. Cell Biochem Biophys. 2013;67(3):837-851.

9. Arthur JC, Gharaibeh RZ, Mühlbauer M, et al. Microbial genomic analysis reveals the essential role of inflammation in bacteria-induced colorectal cancer. Nat Commun. 2014;5:4724.

10. Liu YJ, Zhang S, Hou K, et al. Analysis of key genes and pathways associated with colorectal cancer with microarray technology. Asian Pac J Cancer Prev. 2013;14(3):1819-1823.

11. Barrett T, Wilhite SE, Ledoux P, et al. NCBI GEO: archive for functional genomics data sets - update. Nucleic Acids Res. 2013;41(Database issue):D991-D995.

12. Hong Y, Ho KS, Eu KW, Cheah PY. A susceptibility gene set for early onset colorectal cancer that integrates diverse signaling pathways: implication for tumorigenesis. Clin Cancer Res. 2007;13(4):1107-1114.

13. Gautier L, Cope L, Bolstad BM, Irizarry RA. Affy - analysis of Affymetrix GeneChip data at the probe level. Bioinformatics. 2004; 20(3):307-315.

14. Irizarry RA, Hobbs B, Collin F, et al. Exploration, normalization, and summaries of high density oligonucleotide array probe level data. Biostatistics. 2003;4(2):249-264.

15. Benjamini Y, Hochberg Y. Controlling the false discovery rate: a practical and powerful approach to multiple testing. Journal of the Royal Statistical Society. Series B (Methodological). 1995;57(1): 289-300.

16. Huang da W, Sherman BT, Lempicki RA. Systematic and integrative analysis of large gene lists using DAVID bioinformatics resources. Nat Protoc. 2009;4(1):44-57.

17. Ashburner M, Ball CA, Blake JA, et al. Gene ontology: tool for the unification of biology. The Gene Ontology Consortium. Nat Genet. 2000;25(1):25-29.

18. von Mering C, Huynen M, Jaeggi D, Schmidt S, Bork P, Snel B. STRING: a database of predicted functional associations between proteins. Nucleic Acids Res. 2003;31(1):258-261.
19. Smoot ME, Ono K, Ruscheinski J, Wang PL, Ideker T. Cytoscape 2.8: new features for data integration and network visualization. Bioinformatics. 2011;27(3):431-432.

20. Rhrissorrakrai K, Gunsalus KC. MINE: Module Identification in Networks. BMC Bioinformatics. 2011;12:192.

21. Ely HA, Mellon PL, Coss D. GnRH induces the c-Fos gene via phosphorylation of SRF by the calcium/calmodulin kinase II pathway. Mol Endocrinol. 2011;25(4):669-680.

22. Milde-Langosch K. The Fos family of transcription factors and their role in tumourigenesis. Eur J Cancer. 2005;41(16):2449-2461.

23. Vial E, Sahai E, Marshall CJ. ERK-MAPK signaling coordinately regulates activity of Rac1 and RhoA for tumor cell motility. Cancer Cell. 2003;4(1):67-79.

24. White ES, Muro AF. Fibronectin splice variants: understanding their multiple roles in health and disease using engineered mouse models. IUBMB Life. 2011;63(7):538-546.

25. Gulubova M, Vlaykova T. Immunohistochemical assessment of fibronectin and tenascin and their integrin receptors alpha5beta1 and alpha9betal in gastric and colorectal cancers with lymph node and liver metastases. Acta Histochem. 2006;108(1):25-35.

26. Hanamura N, Yoshida T, Matsumoto E, Kawarada Y, Sakakura T. Expression of fibronectin and tenascin-C mRNA by myofibroblasts, vascular cells and epithelial cells in human colon adenomas and carcinomas. Int J Cancer. 1997;73(1):10-15.

27. Gardina PJ, Clark TA, Shimada B, et al. Alternative splicing and differential gene expression in colon cancer detected by a whole genome exon array. BMC Genomics. 2006;7:325.

28. Vineetha S, Chandra Shekara Bhat C, Idicula SM. Gene regulatory network from microarray data of colon cancer patients using TSK-type recurrent neural fuzzy network. Gene. 2012;506(2):408-416.

29. Lee JH, Lee JS, Kim SE, et al. Tautomycetin inhibits growth of colorectal cancer cells through p21 cip/WAF1 induction via the extracellular signalregulated kinase pathway. Mol Cancer Ther. 2006;5(12):3222-3231.

30. Guengerich FP, Shimada T. Activation of procarcinogens by human cytochrome P450 enzymes. Mutat Res. 1998;400(1-2):201-213.

31. Kumarakulasingham M, Rooney PH, Dundas SR, et al. Cytochrome p450 profile of colorectal cancer: identification of markers of prognosis. Clin Cancer Res. 2005;11(10):3758-3765.

32. Windmill KF, McKinnon RA, Zhu X, Gaedigk A, Grant DM, McManus ME. The role of xenobiotic metabolizing enzymes in arylamine toxicity and carcinogenesis: functional and localization studies. Mutat Res. 1997;376(1-2):153-160.

33. Bethke L, Webb E, Sellick G, et al. Polymorphisms in the cytochrome P450 genes CYP1A2, CYP1B1, CYP3A4, CYP3A5, CYP11A1, CYP17A1, CYP19A1 and colorectal cancer risk. BMC Cancer. 2007;7: 123.

\section{Dovepress}

\section{Publish your work in this journal}

OncoTargets and Therapy is an international, peer-reviewed, open access journal focusing on the pathological basis of all cancers, potential targets for therapy and treatment protocols employed to improve the management of cancer patients. The journal also focuses on the impact of management programs and new therapeutic agents and protocols on patient perspectives such as quality of life, adherence and satisfaction The manuscript management system is completely online and includes a very quick and fair peer-review system, which is all easy to use. Visit http://www.dovepress.com/testimonials.php to read real quotes from published authors. 